\title{
In Situ Observation of Cooling in a Bismuth Telluride and Bismuth-Antimony Telluride Nanoscale Heterojunction
}

Gurleen Bal ${ }^{1}$, Matthew Mecklenburg ${ }^{2}$, William A. Hubbard ${ }^{1}$, Bozo Vareskic ${ }^{1}$, Brian Zutter ${ }^{1}$, Shaul Aloni $^{3}$, and B. C. Regan ${ }^{1}$

1. Department of Physics and Astronomy \& California NanoSystems Institute, University of California, Los Angeles, CA, USA

2. Center for Electron Microscopy and Microanalysis, University of Southern California, Los Angeles, CA, USA

3. Molecular Foundry, Lawrence Berkeley National Laboratory, Berkeley, CA, USA

Thermoelectrics have a wide variety of applications, but their efficiency, typically stated in terms of the figure of merit $Z T$, must be improved before they become economical for non-niche applications. According to theory [1, 2], ZT can be improved relative to the bulk by constructing devices that feature nanometer-scale confinement in one or more dimensions. Here we describe transmission electron microscopy (TEM) observations of cooling in heterojunctions constructed from 2D flakes of exfoliated bismuth telluride and bismuth-antimony telluride.

A 'quintuple' of bismuth telluride consists of five atomic layers and is one nanometer thick [2]. Such thin layers could, in principle, improve $Z T$ by a factor of 13 [1]. Observations have shown ZT enhancement of 30-40\% [2]. In this preliminary work, we report measurements on thermoelectric junctions between many-quintuple layer exfoliated bismuth telluride and bismuth-antimony telluride (see Figure 1). Using scanning TEM electron beam induced current (STEM EBIC) [3], we confirmed the existence of a p-n junction at the interface between two flakes that were overlapped using wettransfer methods.

To investigate the cooling properties of the junctions, we applied plasmon energy expansion thermometry (PEET) [4]. Because the bulk plasmons in bismuth telluride and bismuth-antimony telluride have broad widths, we evaporated indium nanoparticles onto the supporting silicon nitride membrane to serve as local nanothermometers. Applying PEET to one nanoparticle near the heterojunction, we measured this particle's temperature as a function of the reverse-bias junction current (see Figure 2).

The temperature decreased with increasing current magnitude until the junction current $(I)$ reached -60 $\mu \mathrm{A}$. At this point the Joule heating (proportional to $I^{2}$ ) began to dominate the Peltier cooling (proportional to $I$ ). Interpreting the $14 \mathrm{meV}$ shift in the indium plasmon energy in terms of indium's linear thermal expansion coefficient of $3 \times 10^{-5} \mathrm{~K}^{-1}$, we find the device to have cooled by approximately $30 \mathrm{~K}$, which is below the freezing point of water. This performance, good for a single stage cooling device [5], is especially impressive considering the thermal short through the supporting $15 \mathrm{~nm}$-thick silicon nitride membrane. COMSOL modeling of this junction, subject to admittedly large uncertainties, produces smaller temperature shifts than those observed. Further scaling and more complete, quantitative measurements using STEM EBIC and PEET will allow a more definitive determination of the possibilities for significantly improving ZT via nanostructuring such layered materials as bismuth telluride and bismuth antimony telluride. 


\section{References:}

[1] LD Hicks and MS Dresselhaus, Physical Review B 47 (1993), p. 127272.

[2] D Teweldebrhan, V Goyal, and AA Balandin, Nano Letters 10 (2010), p. 1209-1218.

[3] ER White et al, Applied Physics Letters 107 (2016), p. 223104.

[4] M Mecklenburg et al, Science 347 (2015), p. 629-632.

[5] HJ Goldsmid, Introduction to Thermoelectricity, ed. R Hull et al, (Springer, New York) p.17.

[6] This work was supported by FAME, one of six centers of STARnet, a Semiconductor Research Corporation program sponsored by MARCO and DARPA, by National Science Foundation (NSF) award DMR-1611036, and by NSF STC award DMR-1548924. Data presented were acquired at the Center for Electron Microscopy and Microanalysis at the University of Southern California.
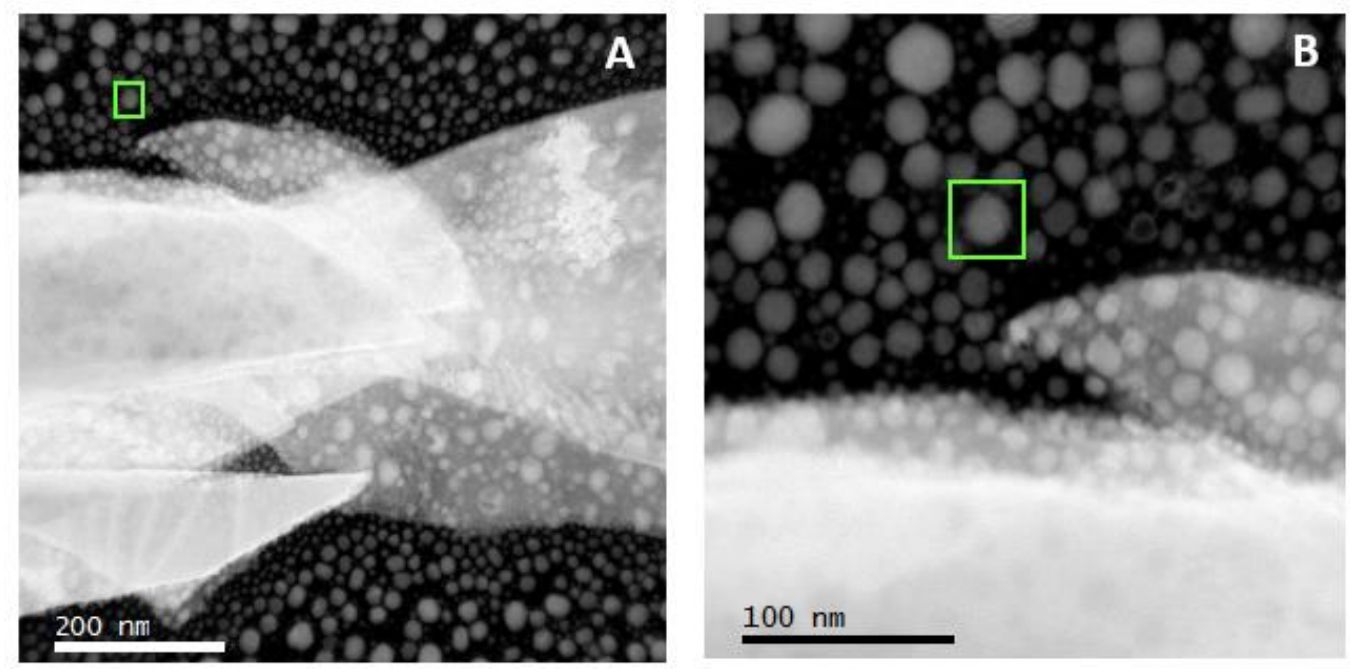

Figure 1. A) STEM image of a thermoelectric device (p-type $\mathrm{Bi}_{x} \mathrm{Sb}_{2-x} \mathrm{Te}_{3}$ (left) and n-type $\mathrm{Bi}_{2} \mathrm{Te}_{3}$ (right)) on a $15 \mathrm{~nm}$ thick silicon nitride membrane. B) A higher magnification STEM image of indium nanoparticles near the device. Green box indicates the nanoparticle over which PEET was applied.
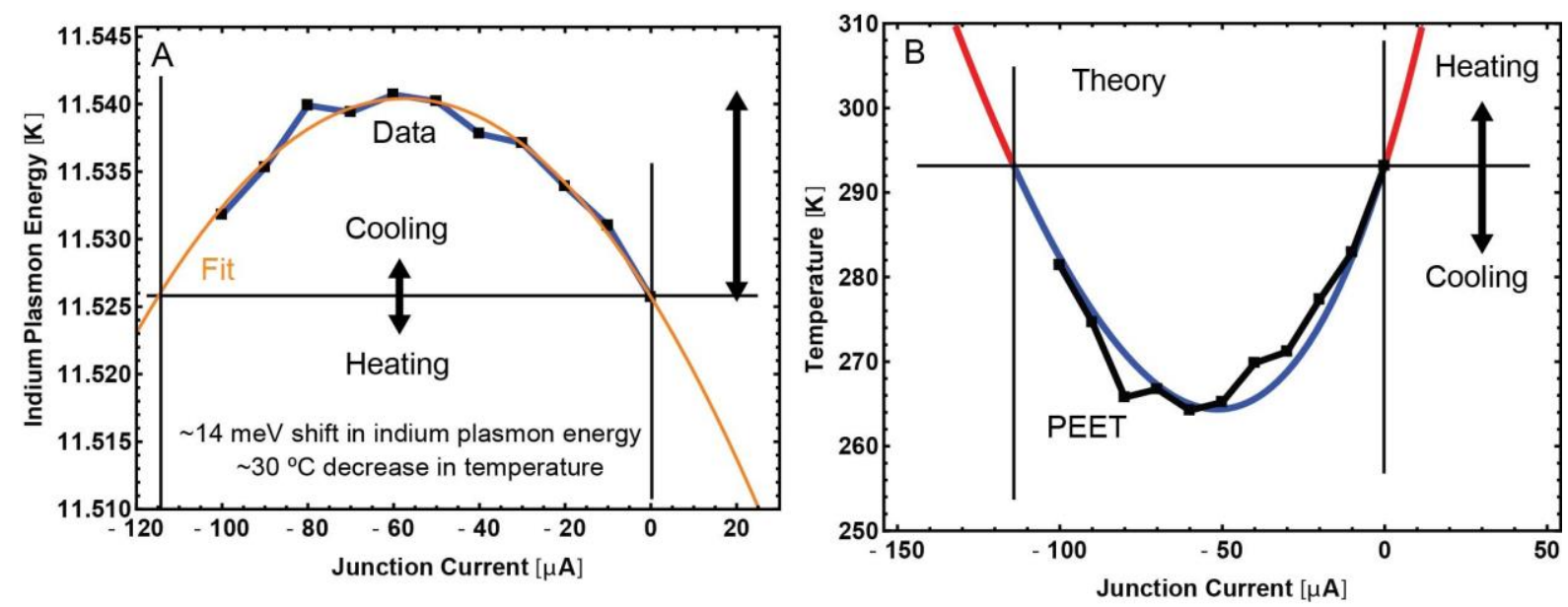

Figure 2. A) The sampled indium nanothermometer's measured plasmon energy shifts as a function of heterojunction current. B) The corresponding temperature shifts, along with simulation results showing the expected heating and cooling behavior. 